\title{
Perfil lipídico da polpa e amêndoa da guarirova
}

\author{
Nutritional quality of oil and almond guarirova pulp
}

\author{
Vanessa Taís Nozaki ${ }^{I}$ Cláudia Leite Munhoz ${ }^{\mathrm{I}}$ Rita de Cássia Avellaneda Guimarães ${ }^{\mathrm{I}}$ \\ Priscila Aiko Hiane ${ }^{\mathrm{I}}$ Maxwell Parrela AndreuII Luiz Henrique Viana ${ }^{\text {II }}$ \\ Maria Lígia Rodrigues Macedo ${ }^{\mathrm{I}^{*}}$
}

\section{RESUMO}

Os óleos de frutos estão sendo estudados por seu potencial na indústria de alimentos e também para aumento do consumo de ácidos graxos monos e poli-insaturados. Este estudo objetivou determinar as constantes físico-químicas, composição centesimal, identificar e quantificar os principais ácidos graxos presentes na fração lipídica da polpa e da amêndoa dos frutos de guarirova, espécie Syagrus oleracea (Mart.) Becc. Foi realizada a caracterização físico-química dos óleos, composição de ácidos graxos e índices de qualidade nutricional. O óleo da amêndoa possui maior concentração de ácidos graxos saturados $(89,2 \%)$, sendo representado principalmente pelo ácido láurico (48,34\%). A polpa apresentou ácidos graxos monoinsaturados (24,84\%) e poliinsaturados $(33,25 \%)$, com maior percentual de ômega-6 (31,94\%) e ômega-9 (19,15\%); e menor de ômega-3 (1,31\%).

Palavras-chave: ácidos graxos, lipídios, guarirova.

\section{ABSTRACT}

The oils of fruits are being studied for its potential in the food industry and also to increase the consumption of mono and polyunsaturated fatty acids. This study aimed to determine the physio-chemical constants, chemical composition and identify and quantify the major fatty acids present in the lipid fraction of pulp and kernel of the fruits of guarirova, species Syagrus oleracea (Mart.) Becc. It was performed the physicochemical characterization of oils, fatty acid composition and nutritional quality indexes. Almond oil has the highest concentration of saturated fatty acids $(89,2 \%)$, represented mainly by lauric acid (48,34\%). The pulp showed monounsaturated $(24,84 \%)$ and polyunsaturated fatty acids (33,25\%), with higher percentage of omega-6 (31,94\%) and omega-9 $(19,15 \%)$ and lower omega-3 $(1,31 \%)$.

Key words: fatty acids, lipids, guarirova.

\section{INTRODUÇÃO}

Os óleos de frutos nativos do Cerrado, como, por exemplo, a bocaiuva, e as sementes comestíveis regionais, como o baru, estão sendo estudados devido a sua importância e aplicabilidade em indústria de alimentos e na saúde. Os óleos e gorduras são bastante utilizados na alimentação, sendo que $80 \%$ da sua produção mundial possuem essa finalidade (LAURELES et al., 2002).

$\mathrm{Na}$ indústria de alimentos, existe a necessidade de pesquisas quanto às novas fontes de lipídios que não apresentem na sua formulação gorduras trans (TARRAGO-TRANI et al., 2003), pois, em produtos alimentícios, possuem como ingrediente a gordura vegetal hidrogenada, rica em ácidos graxos trans. Este tipo de gordura pode inibir o metabolismo de ácidos graxos essenciais, aumentar os níveis de lipoproteína de baixa densidade (LDL) e diminuir lipoproteína de alta densidade (HDL), dessa maneira seu consumo pode ser prejudicial à saúde (COSTA et al., 2006).

Em relação à saúde, pode-se destacar a importância do consumo de ácidos graxos mono e poliinsaturados para melhorar o perfil de lipídio sérico. Estudos são necessários para descobrir novas fontes de ácidos graxos insaturados e utilizá-los como suplementação na alimentação humana (FREITAS \& NAVES, 2010).

\footnotetext{
IDepartamento de Tecnologia de Alimentos e Saúde Pública, Universidade Federal de Mato Grosso do Sul (UFMS), 79070-900, Campo Grande, MS, Brasil. E-mail: bioplant@terra.com.br. *Autor para correspondência.

IILaboratório de Combustíveis, Departamento de Química, UFMS, Campo Grande, MS, Brasil.
} 
Pesquisas sobre alguns frutos do Cerrado demonstraram elevados teores de lipídios, contendo ácidos graxos saturados e insaturados (SILVA et al., 2001). Já existem trabalhos mostrando esse perfil em frutos do Cerrado, como a bocaiuva (HIANE et al., 2005), em que na polpa encontrou-se $25,52 \%$ de ácidos graxos saturados, $66,88 \%$ de monoinsaturados e $7,62 \%$ de poli-insaturados e no baru foram encontrados $22,92 \%$ de ácidos graxos saturados e 75,58\% de insaturados (VERA et al., 2009).

A guarirova, por sua vez, é um fruto do bioma Cerrado e que necessita de estudos para conhecimento de sua composição nutricional e perfil lipídico. A guarirobeira é uma palmeira (Syagrus oleracea (Mart.) Becc.) do Cerrado que possui frutos em cachos, de coloração verde-amarelada, com uma amêndoa branca oleaginosa comestível (LORENZI et al., 2004).

Apesar do consumo regional pela população local da polpa e da amêndoa in natura de guarirova, o fruto também é aplicado na gastronomia, no entanto pouco se conhece da composição de ácidos graxos. Diante do exposto, o presente estudo objetivou determinar as constantes físico-químicas, identificar e quantificar os principais ácidos graxos presentes na fração lipídica da polpa e da amêndoa dos frutos de guarirova, espécie Syagrus oleracea (Mart.) Becc.

\section{MATERIAL E MÉTODOS}

Matéria-prima

Frutos maduros da guarirova (Syagrus oleracea (Mart.) Becc.) foram coletados na cidade de Campo Grande no Estado de Mato Grosso do Sul, em fevereiro de 2010. Os frutos foram higienizados, descascados e despolpados manualmente. A polpa do fruto foi dessecada em Estufa de Circulação Forçada de $\operatorname{Ar}$ (LAWES) $\mathrm{a} 45^{\circ} \mathrm{C}$ por aproximadamente 8 h. A amêndoa foi retirada do fruto quebrando-se o tegumento. A polpa dessecada e a amêndoa foram trituradas separadamente em triturador Turratec e tamisadas a 60 mesh, baseado no estudo de HIANE et al. (2006).

Caracterização físico-química dos óleos

Os óleos da polpa e da amêndoa da guarirova foram extraídos a frio de acordo com a metodologia de BLIGH \& DYER (1959). O índice de refração foi obtido por leitura direta em refratômetro de Abbé (TecnaL/ RL3). O conteúdo de iodo foi determinado pelo método de Wijs, utilizando-se solução de Wijs e $0,1 \mathrm{~mol} \mathrm{~L}^{-1}$ de tiossulfato de sódio. Para determinar o índice de saponificação, utilizou-se solução de $4 \%$ de hidróxido de potássio e titulação com $0,5 \mathrm{~N}$ de ácido clorídrico (BRASIL, 2005).
Composição dos ácidos graxos

Para a análise de composição de ácidos graxos, a fração lipídica total foi submetida à saponificação com $\mathrm{KOH}$ metanólico, seguida de esterificação com mistura de $\mathrm{H}_{2} \mathrm{SO}_{4}$ e NH $\mathrm{NH}_{4} \mathrm{Cl}$ em metanol e transferida para hexano, segundo método modificado por MAIA \& RODRIGUEZ-AMAYA(1993). A análise dos ésteres metílicos dos ácidos graxos foi realizada em cromatógrafo gasoso (Varian, modelo Star 3400), equipado com detector de ionização de chama, injetor Split/splitless, coluna capilar de sílica fundida contendo polietilenoglicol como fase estacionária (DBWax, 30mx0,25mm, J\&W Scientific), nas seguintes condições cromatográficas: temperatura do injetor $250^{\circ} \mathrm{C}$; temperatura da coluna $180^{\circ} \mathrm{C}$ durante 20 minutos, programada a $2^{\circ} \mathrm{C}$ por minuto até $220^{\circ} \mathrm{C}$; temperatura do detector $260^{\circ} \mathrm{C}$; gás de arraste hidrogênio com fluxo de $1,1 \mathrm{~mL} \mathrm{~min}^{-1}$; gás make-up nitrogênio a $22 \mathrm{~mL} \mathrm{~min}^{-1}$; e volume de injeção de $0,5 \mu \mathrm{L}$. Para a identificação dos ácidos graxos, compararam-se os tempos de retenção com os dos padrões ésteres metílicos (Sigma-Aldrich), enquanto a quantificação foi realizada pela normalização de área, expressando-se o resultado em percentual de área de cada ácido sobre a área total de ácidos graxos (\%). A identificação dos picos foi realizada com base no tempo de retenção e na comparação com padrões de ésteres metílicos de ácidos graxos, a quantificação usando os fatores de correção de área de acordo a norma Ce 1e-91 da AMERICAN OIL CHEMISTS SOCIETY (AOCS) (1998).

Índices da fração lipídica

A fração lipídica foi avaliada através do Índice de Aterogenicidade (IA) (1), Índice de Trombogenicidade (IT) (2), segundo ULBRICHT \& SOUTHGATE (1991); e razão entre ácidos graxos hipocolesterolêmicos e hipercolesterolêmicos (HH) (3), segundo SANTOS-SILVA et al. (2002), utilizando-se os dados da composição em ácidos graxos.

(1) $I A=\frac{C_{12: 0}+4 \times C_{14: 0}+C_{16: 0}}{\Sigma A G M I+\Sigma \omega 6+\Sigma \omega 3}$

(2) $I T=\frac{C_{14: 0}+C_{16.0}+C_{18: 0}}{0.5 \times \Sigma A G M I+0.5 \times \Sigma \omega 6+3 \times \Sigma \omega 3}$

(3) $H H=\frac{C_{18: 1 C I 59}+C_{18: 2 \omega 6}+C_{20: 4 \omega 6}+C_{18: 3 \omega 3}+C_{20.5 \omega 3}+C_{22: 5 \omega 3}+C_{22.6 \omega 3}}{C_{14: 0}+C_{16: 0}}$

Onde, AGMI são os ácidos graxos monoinsaturados.

Análise estatística

Os valores da composição de ácidos graxos foram submetidos à análise de variância (ANOVA). O teste de Tukey foi aplicado para as variâncias desiguais entre os valores médios das amostras, mantendo-se o nível de significância de 5\% em todas as análises. 


\section{RESULTADOS E DISCUSSÃO}

Analisando-se as características dos óleos, pode-se observar que o índice de refração encontrado foi de $1,470 \pm 0,01$ e 1,458 $\pm 0,02$ na polpa e amêndoa, respectivamente, o índice de iodo $49,93 \pm 2,91$ na polpa e 7,34 $\pm 0,61\left(\mathrm{~g} \mathrm{I}_{2} 100 \mathrm{~g}_{\text {óleo }}{ }^{-1}\right)$ na amêndoa e ainda índice de saponificação na polpa $225,56 \pm 1,65$ e na amêndoa 278,05 $\pm 2,84$ (mg KOH g óleo ${ }^{-1}$ ). Em comparação com estudo realizado por BORA \& MOREIRA (2003), o óleo da amêndoa da guarirova no estado da Paraíba obteve o índice de refração $(1,444 \pm 0,01)$ e de saponificação $(226,0 \pm 2,8)$, sendo que os resultados foram semelhantes. No entanto, houve diferença para o índice de iodo $(27,4 \pm 1,3)$. Esse índice proporciona uma medida do grau de insaturação das gorduras extraídas por éter. Por essa razão, quanto maior a insaturação de um ácido graxo, maior será a sua capacidade de absorção de iodo e, consequentemente, maior será o índice de iodo.

Quanto à identificação e quantificação dos ácidos graxos (Tabela 1), a amêndoa possui maior concentração de ácidos graxos saturados $(89,2 \%)$ com
48,34\% de ácido láurico (C12:0). No Brasil, os óleos de coco, palmiste e babaçu, normalmente, são as principais fontes deste ácido graxo (MACHADO et al.,2006). Esse ácido graxo pode oferecer benefícios à saúde, sendo responsável por algumas funções como, por exemplo, ação antimicrobiana (LOGRADA et al., 2010); função de melhorar o sistema imunológico (WEATHERILL et al., 2005), além de ser anti-inflamatório (MENENDÉZ et al., 2006). Entretanto, é importante ressaltar que os estudos ainda não são conclusivos e que ainda não existe recomendação nutricional para seu consumo. $\mathrm{O}$ ácido graxo láurico também possui aplicabilidade na indústria de alimentos em substituição à gordura vegetal hidrogenada, que possui elevada concentração de gordura trans (FARIA et al., 2008).

Apesar dos benefícios à saúde, citados acima, quando usado na alimentação humana, o ácido graxo láurico é um dos principais ácidos graxos saturados responsáveis pela elevação sérica de colesterol (SANTOS et al., 2001).

Além dos ácidos graxos saturados, foram também identificados os ácidos graxos monoinsaturados

Tabela 1 - Composição dos ácidos graxos dos óleos da polpa e amêndoa de guarirova (Syagrus oleracea (Mart.) Becc.) ${ }^{1}$

\begin{tabular}{|c|c|c|}
\hline Ácidos graxos & Óleo da polpa (\%) & Óleo da amêndoa (\%) \\
\hline Ácido hexanoíco (C6:0) & $0,21 \pm 0,01^{\mathrm{a}}$ & $0,48 \pm 0,01^{\text {a }}$ \\
\hline Ácido caprílico (C8:0) & $0,54 \pm 0,02^{\text {a }}$ & $10,30 \pm 0,12^{\mathrm{b}}$ \\
\hline Ácido cáprico (C10:0) & $0,30 \pm 0,01^{\mathrm{a}}$ & $6,65 \pm 0,06^{\mathrm{b}}$ \\
\hline Ácido undecanoico (C11:0) & n.d. & $0,04 \pm 0,01$ \\
\hline Ácido láurico (C12:0) & $0,87 \pm 0,02^{\mathrm{a}}$ & $48,34 \pm 0,18^{\mathrm{b}}$ \\
\hline Ácido tridecanoico (C13:0) & n.d. & $0,05 \pm 0,02$ \\
\hline Ácido mirístico (C14:0) & $0,83 \pm 0,02^{\mathrm{a}}$ & $14,34 \pm 0,07^{\mathrm{b}}$ \\
\hline Ácido pentadecanoico (C15:0) & $0,03 \pm 0,01$ & n.d. \\
\hline Ácido palmítico (C16:0) & $36,50 \pm 0,15^{\mathrm{a}}$ & $5,14 \pm 0,05^{\mathrm{b}}$ \\
\hline Ácido heptadecanoico (C17:0) & $0,06 \pm 0,02$ & n.d. \\
\hline Ácido esteárico (C18:0) & $1,12 \pm 0,01^{\mathrm{a}}$ & $3,72 \pm 0,07^{b}$ \\
\hline Ácido araquídico (C20:0) & $0,22 \pm 0,01^{\mathrm{a}}$ & $0,10 \pm 0,01^{\mathrm{a}}$ \\
\hline Ácido behênico (C22:0) & $0,09 \pm 0,01$ & n.d \\
\hline Ácido tetracosanoico (C24:0) & $0,28 \pm 0,01^{\mathrm{a}}$ & $0,04 \pm 0,02^{\mathrm{a}}$ \\
\hline$\Sigma$ SATURADOS (SFA) & 41,05 & 89,2 \\
\hline Ácido palmitoleico $(\mathrm{C} 16: 1 \omega 7)$ & $1,52 \pm 0,02$ & n.d. \\
\hline Ácido vacênico (C18:1@7) & $4,17 \pm 0,01$ & n.d \\
\hline Ácido oleico $(\mathrm{C} 18: 1 \omega 9)$ & $19,01 \pm 0,07^{\mathrm{a}}$ & $7,98 \pm 0,13^{b}$ \\
\hline Ácido gadoleico $(\mathrm{C} 20: 1 \omega 9)$ & $0,12 \pm 0,01$ & n.d. \\
\hline Ácido erucico (C22:1 $\omega 9)$ & $0,02 \pm 0,02$ & n.d. \\
\hline$\Sigma$ MONOINSATURADOS (MUFA) & 24,84 & 7,98 \\
\hline 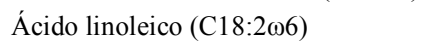 & $31,92 \pm 0,09^{\mathrm{a}}$ & $1,63 \pm 0,03^{\mathrm{b}}$ \\
\hline Ácido alfa-linolênico (C18:3 103$)$ & $1,31 \pm 0,01$ & n.d. \\
\hline 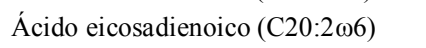 & $0,02 \pm 0,02$ & n.d. \\
\hline$\Sigma$ POLI-INSATURADOS (PUFA) & 33,25 & 1,63 \\
\hline
\end{tabular}

${ }^{1}$ Dados apresentados como média \pm desvio-padrão. Letras iguais na mesma linha não diferem entre si $(\mathrm{P}>0,05)$. $\mathrm{Nd}$ : não detectado.

Ciência Rural, v.42, n.8, ago, 2012. 
(MUFA) e poli-insaturados (PUFA). A amêndoa da guarirova possui $7,98 \%$ de ácido oleico e 1,63\% de linoleico. E a polpa apresentou maior conteúdo percentual de MUFA e PUFA em relação à amêndoa.

Arelação MUFA/SFAEPUFA/SFA(Tabela 2), na polpa, foi de 0,60 e 0,80 respectivamente; observouse também que a quantidade de ácidos graxos poliinsaturados foi maior na polpa. Valores dessa relação menores que 0,45 são considerados indesejáveis, pois podem aumentar o colesterol sanguíneo (DEPARTMENT OF HEALTH AND SOCIAL SECURITY, 1994). Apenas a polpa da guarirova obteve valor acima de 0,45 .

$\mathrm{Na}$ polpa, foram encontrados valores percentuais de $41,05 \%$ de ácidos graxos saturados, sendo que, destes, $36,50 \%$ são de ácido palmítico (C16:0). Dos 24,84\% de MUFA, 19,01\% estão representados pelo ácido oleico (C18:1 199$)$. Os PUFA representam $33,25 \%$ do total de ácidos graxos, sendo $31,92 \%$ de ácido linoleico(C18:2 $\omega 6)$. O alto conteúdo de ácido linoleico é nutricionalmente importante, pois é um ácido graxo essencial (LUZIA \& JORGE; 2009), precursor dos demais ácidos graxos da família ômega6 , sendo que este juntamente com o alfa-linolênico (C18:3w3) sao necessários para as funções cerebrais e os impulsos nervosos (SPOSITO et al., 2007).

O consumo de alimentos com MUFAe PUFA é importante para a diminuição das frações lipídicas de LDL (Lipoproteína de Baixa Densidade) e VLDL (Lipoproteína de Muito Baixa Densidade). Entretanto, quando consumidos em excesso, podem induzir maior oxidação lipídica e diminuir o HDL (Lipoproteína de Alta densidade) (SPOSITO et al., 2007).

Tabela 2 - Índices da fração lipídica dos óleos da polpa e da amêndoa da guarirova (Syagrus oleracea (Mart.) Becc.).

\begin{tabular}{lcc}
\hline Índices & Óleo da polpa & Óleo da amêndoa \\
\hline MUFA/SFA & 0,60 & 0,08 \\
PUFA/SFA & 0,80 & 0,01 \\
$\omega-6 / \omega-3$ & 24,38 & - \\
IA & 0,69 & 11,53 \\
IT & 1,32 & 4,82 \\
HH & 1,39 & 0,49 \\
\hline
\end{tabular}

SFA: Saturated fatty acids. MUFA: Monounsaturated fatty acids. PUFA: Polyunsaturated fatty acids. MUFA/SFA: relação entre ácidos graxos monoinsaturados e saturados. PUFA/SFA: relação entre ácidos graxos poli-insaturados e saturados. $\omega-6 / \omega-3$ : relação entre ácidos graxos $\omega-6$ e $\omega-3$. IA: índice de aterogenecidade. IT índice de trombogenecidade. HH: razão entre ácidos graxos hipocolesterolêmicos e hipercolesterolêmicos. Em que $\omega$ : ômega.
Concentrações elevadas de ácido oleico (presente, por exemplo, no azeite de oliva e abacate) e linoleico são características de nozes e sementes comestíveis, entretanto em polpa de frutas este fato possui menor ocorrência (FREITAS \& NAVES, 2010). O ácido oleico (C18:1 $\omega 9)$ e um ácido graxo monoinsaturado que não promove oxidação lipídica e nem diminui o HDL, no entanto, melhora a oxidação de LDL. Esse ácido graxo é recomendado em dietas, pois exerce funções de proteção contra aterogênese (SPOSITO et al., 2007).Comparando-se as concentrações de ácidos graxos entre a polpa e amêndoa (Tabela 1), observou-se que a polpa possui maior teor $(\mathrm{P}<0,05)$ para a maioria dos ácidos graxos identificados, com exceção de C6:0, C20:0 e C24:0 que obtiveram resultados semelhantes, ficando com o $\mathrm{P}>0,05$.

As dietas devem apresentar a razão de ômega6/ômega-3 entre 4:1 ou 5:1, a fim de reduzir o risco de prevalência de doenças crônicas (SIMOPOULOS, 2006). O DEPARTMENT OF HEALTH AND SOCIAL SECURITY (1994) também sugeriu que o balanço entre os ácidos graxos poli-insaturados ômega-6/ômega-3 da dieta não deve exceder 4,0 . No óleo da polpa da guarirova, encontrou-se a proporção de $24: 1$, semelhante ao que ocorre no milho $(32,5: 1)$ e na aveia $(22: 1)$ (MARTIN et al., 2006). Esses valores estão acima da recomendação e não são indicados na práxis alimentar diária. É importante salientar que os indivíduos devem consumir alimentos ricos em $\omega-3$ para diminuir a relação ômega-6/ômega-3 na alimentação diária como agente protetor para doenças cardiovasculares (MARTIN et al., 2006). Segundo SIMOPOULOS (2006), diversas doenças estão associadas ao aumento do consumo de ômega-6 e diminuição do ômega-3, como, câncer, diabetes, asma, sendo importante o equilíbrio do consumo entre esses ácidos graxos.

Segundo as recomendações do INSTITUTE OF MEDICINE (2005), o consumo diário de 100g de polpa de guarirova atenderia $41,17 \%$ das necessidades de ômega- 6 de crianças ( 7 a 10 anos), 26,50\% de adultos e idosos de ambos os gêneros, $18 \%$ das necessidades de ômega 3 em crianças ( 7 a 10 anos) e 11,04\% de adultos e idosos do gênero masculino e feminino. Para consumir $100 \mathrm{~g}$ de polpa do fruto, são necessários de sete a oito frutos, já que cada fruto tem em média $13 \mathrm{~g}$ de polpa.

Segundo ASSUNÇÃO (2007), os pesquisadores ULBRICHT \& SOUTHGATE (1991) propuseram dois índices que avaliam os ácidos graxos e seus efeitos no metabolismo das lipoproteínas. Esses índices chamados de IA (Índice de Aterogenicidade) e IT (Índice de Trombogenicidade) são aplicados para avaliar a qualidade nutricional dos óleos. Não existem 
valores recomendados para estes índices, no entanto, valores menores exprimem uma relação de ácidos graxos mais favoráveis e os valores maiores sugerem que o consumo do óleo estudado traz malefícios á saúde.

Os índices de qualidade nutricional da fração lipídica das amostras avaliadas, o IA, IT e HH (Tabela 2), foram superiores nos óleos da polpa ( $\mathrm{IA}=0,69 ; \mathrm{IT}=1,32$; $\mathrm{HH}=1,39)$ do que da amêndoa ( $\mathrm{IA}=11,53 ; \mathrm{IT}=4,82$; $\mathrm{HH}=0,49)$. RAMOS-FILHO et al. (2008) encontraram índices mais elevados de $\mathrm{HH}$ em peixes, como, por exemplo, 1,84 para pintado e ainda para IA e IT encontraram 0,49 e 0,33 , respectivamente. No óleo de coco, foram encontrados IA=14 e IT=6(ULBRICHT \& SOUTHGATE, 1991), sendo valores mais próximos do óleo da amêndoa da guarirova.

\section{CONCLUSÃO}

As constantes físico-químicas das amostras avaliadas demonstraram que os índices de refração e saponificação estavam semelhantes a outro estudo realizado com a guarirova e o índice de iodo apresentou-se menor.

A amêndoa possui maior concentração de gordura saturada com predomínio do ácido graxo láurico. Não foram identificados ácidos graxos do tipo ômega-3 e observaram-se quantidades pequenas de ômega-6 e ômega-9. A polpa apresentou concentrações acima de 50\% de ácidos graxos monoinsaturados e poliinsaturados, com maior percentual de ômega-6 e ômega9; e menor de ômega-3.

Em relação aos índices de qualidade nutricional, o óleo da polpa da guarirova apresentou resultados melhores em relação ao da amêndoa.

\section{AGRADECIMENTO}

À Coordenação de Apoio a Pesquisa de Ensino Superior (CAPES), pela bolsa de doutorado concedida.

\section{REFERÊNCIAS}

AMERICAN OIL CHEMISTS SOCIETY - AOCS. Official methods and recommended practices of the AOCS. 5 ed. Champaign, 1998. $1430 \mathrm{p}$.

ASSUNÇÃO, J.M.P. Contribuição para o estudo da composição lipídica e do valor nutricional de leite e produtos lácteos dos Açores. 2007. 113f. Dissertação (Mestrado em Controlo de Qualidade e Toxicologia dos Alimentos) - Curso de Pós-graduação em Controlo de Qualidade e Toxicologia dos Alimentos, Universidade de Lisboa.

BLIGH, E.G.; DYER, W.J. A rapid method of total lipid extraction and purification. Canadian. Journal Biochemistry and Physiology, v.37, n.8, p.911-917, 1959. Disponível em: $<$ http://rparticle.web.cisti.nrc.ca/rparticle/
AbstractTemplateServlet?calyLang=eng\&journal $=$ cjpp\&volume $=$ $37 \&$ year $=1959 \&$ issue $=8 \&$ msno $=y 59-099>$. Acesso em: 10 nov. 2010. doi: 10.1139/y59-099.

BORA, P. S.; MOREIRA, R.V.R. Catolé palm (Syagrus oleracea Mart) fruits: fatty and amino acids composition. Grasas y Aceites, v.54, n.2, p.145-150, 2003. Disponível em: <http:/ /grasasyaceites.revistas.csic.es/index.php/grasasyaceites/article/ download/257/258/>. Acesso em: 06 dez. 2009. doi: 10.3989/ gya.2003.v54.i2

BRASIL. Ministério da Saúde. Agência Nacional de Vigilância Sanitária (ANVISA). Métodos físico-químicos para análise de alimentos. Brasília: Ministério da Saúde, 2005. 1018p.

COSTA, A.G.V. et al. Ácidos graxos trans: alimentos e efeitos na saúde. ALAN-Archivos latinoamericano de Nutricion, v.56, n.1, p.12-21, 2006. Disponível em: <http://www.alanrevista.org/ ediciones/2006-1/acidos_graxos_trans.asp>. Acesso em: 10 abr. 2011.

DEPARTMENT OF HEALTH AND SOCIAL SECURITY. Nutritional aspects of cardiovascular disease. London: HMSO, 1994. 178 p. (Report on Health and Social Subjects, n.46).

FARIA, J.P. et al. Caracterização química da amêndoa de coquinho-azedo (Butia capitata var capitata). Revista Brasileira de Fruticultura, v.30, n.2, p.549-552, 2008. Disponível em: <http://www.scielo.br/ scielo.php?script=sci arttext\&pid=S010029452008000200049>. Acesso em: 12 jan. 2010. doi: 10.1590/S0100-29452008000200049.

FREITAS, B.F.; NAVES, M.M.V. Composição química de nozes e sementes comestíveis e sua relação com a nutrição e saúde. Revista de Nutrição, v.23, n.2, p.269-279, 2010. Disponível em: $<$ http://www.scielo.br/pdf/rn/v23n2/v23n2a10.pdf $>$. Acesso em: 23 fev. 2010. doi: 10.1590/S1415-52732010000200010.

HIANE, P.A. et al. Chemical and nutrition evaluation of kernels of bocaiuva, Acrocomia aculeata (Jacq.) Lodd. Ciência e Tecnologia de Alimentos, v.26, n.3, p.683-689, 2006. Disponível em: <http:/ /www.scielo.br/scielo.php?script=sci_arttext\&pid=S0101$20612006000300031 \mathrm{nnn}>$. Acesso em: 10 dez. 2011. doi: 10.1590/ S0101-20612006000300031.

HIANE, P.A. et al. Óleo da polpa e amêndoa de bocaiúva, Acrocomia aculeata (jacq.) lodd. Caracterização e composição em ácido graxos. Brazilian Journal of Food Technology, v.8, n.3, p.256-259, 2005. Disponível em:<http:// www.ital.sp.gov.br/bj/artigos/bjft/2005/bjtf_71_04.pdf $>$. Acesso em: 28 nov. 2010 .

INSTITUTE OF MEDICINE. Dietary reference intakes for energy, carbohydrate, fiber, fat, fatty acids, cholesterol, protein and amino acids. Washington: National Academy, 2005. 1357p.

LAURELES, L.R. et al. Variability in fatty acid and triacylglycerol composition of the oil of coconut (Cocos nucifera L.) hybrids and their parentals. Journal of Agricultural and Food Chemistry, v.50, p.1581-1586, 2002. Disponível em: <http://www.medscape.com/medline/ abstract/11879040>. Acesso em: 30 mar. 2010.

LOGRADA, T. et al. Chemical composition and antimicrobial activity of essential oils of Genista ulicina and $\boldsymbol{G}$ vepres. Natural Product Communications, v.5, n.5, p.835-838, 2010. Disponível em: $<$ http:http://pubget.com/search?q=authors\%3A\%22Hafsa+Silini\%22>. Acesso em: 15 fev. 2011.

Ciência Rural, v.42, n.8, ago, 2012. 
LORENZI, H. et al. Palmeiras brasileiras e exóticas cultivadas. Nova Odessa: Plantarum, 2004. 432p.

LUZIA, D.M.M.; JORGE, N. Composição centesimal, potencial antioxidante e perfil dos ácidos graxos de sementes de jambolão (Syzygium cumini L.). Revista Ciência Agronômica, v.40, n.2, p.219-223, 2009. Disponível em: <http:// www.ccarevista.ufc.br/seer/index.php/ccarevista/article/view/ 514>. Acesso em: 20 fev. 2010

MACHADO, G.C. et al. Composição em ácidos graxos e caracterização física e química de óleos hidrogenados de coco babaçu. Revista Ceres, v.53, n.308, p.463-470, 2006 Disponível em: <http:www.ceres.ufv.br/CERES/revistas/ V53N308P06506.pdf>. Acesso em: 27 mar. 2010.

MAIA, E.L.; RODRIGUEZ-AMAYA, D.B. Avaliação de um método simples e econômico para a metilação de ácidos graxos com lipídios de diversas espécies de peixes. Revista do Instituto Adolfo Lutz, v.53, n.1/2, p.27-35, 1993

MARTIN, C.A. et al. Ácidos graxos poli-insaturados ômega-3 e ômega-6: importância e ocorrência em alimentos. Revista de Nutrição, v.19, n.6, p.761-770, 2006. Disponível em: $<$ http://www.scielo.br/scielo.php?pid=s 1415 $52732006000600011 \&$ script $=$ sci_arttext $>$. Acesso em: 20 maio, 2010. doi: 10.1590/S1415-52732006000600011.

MENENDÉZ, R. et al. Efectos del D-004, Extracto Lipídico de los Frutos de la Palma Real (Roystonea regia), sobre el Granuloma inducido por Algodón en Ratas y sobre la Lipoxigenasa presente en Leucocitos Polimorfonucleares (PMNs). Acta Farmacéutica Bonaerense, v.25, n.2, p.213218, 2006. Disponível em: <http:www.latamjpharm.org/.../2/ LAJOP_25_2_1_8_599W506H1E.pdf>. Acesso em: 21 jan. 2010. doi: 10.1038 /aja.2008.34

RAMOS-FILHO, M.M. et al. Perfil lipídico de quatro espécies de peixes da região pantaneira de Mato Grosso do Sul. Ciência e Tecnologia de Alimentos, v.28, n.2, p.361-365, 2008. Disponível em: <http://www.scielo.br/scielo.php?pid=S0101$20612008000200014 \&$ script $=\mathrm{sci}$ abstract\&tlng=pt $>$. Acesso em: 11 jan. 2010. doi: 10.1590/S0101-20612008000200014.

SANTOS D.R. et al. III Diretrizes brasileiras sobre dislipidemias e diretrizes de Prevenção da Aterosclerose da Sociedade Brasileira de Cardiologia. Arquivos Brasileiros de Cardiologia, v.77, n.3, p.1-48, 2001. Disponível em: <http: 189.28.128.100/ dab/docs/publicacoes/geral/diretrizcorreta.pdf $>$. Acesso em: 13 fev. 2010. doi: 10.1590/S0066-782X2001001500001.
SANTOS-SILVA J. et al. Effect of genotype, feeding system and slaughter weigt on the quality of light lambs. II. Fatty acid composition of meat. Livestock Production Science, v.77, n.2/3, p.187-194, 2002. Disponível em: <http:// www.journals.elsevierhealth.com/periodicals/livest/article/ S0301-6226(02)00059-3/fulltext>. Acesso em: 30 nov. 2010. doi: 10.1016/S0301-6226(02)00059-3

SILVA, D.B. et al. Frutas do cerrado. Brasília: Embrapa Informação Tecnológica, 2001. 178p.

SIMOPOULOS, A.P. Evolutionary aspects of diet, the omega6/omega-3 ratio and genetic variation: nutritional implications for chronic diseases. Biomedicine \& Pharmacotherapy, v.60, n.9, p.502-507, 2006. Disponível em: <http://www.emconsulte.com/article/148991>. Acesso em: 20 jan. 2010. doi: 10.1016/j.biopha.2006.07.080.

SPOSITO, A.C. et al. IV Diretriz Brasileira sobre Dislipidemias e Prevenção da Aterosclerose: Departamento de Aterosclerose da Sociedade Brasileira de Cardiologia. Arquivos Brasileiros de Cardiologia, v.88, n.1, p.2-19, 2007. Disponível em:<http://www.scielo.br/ scielo.php?script $=$ sci_arttext\&pid $=$ S0066782X2007000700001 $\& \operatorname{lng}=$ pt\&nrm=iso\&tlng=pt $>$. Acesso em: 12 maio, 2010. doi: 10.1590/S0066-782X2007000700001.

TARRAGO-TRANI, M.T. et al. New and existing oils fats used in products with reduced trans-fatty acid content. Journal of the American, n.106, n.6, p.867-880, 2003. Disponível em: $<$ http://www.adajournal.org/article/S0002-8223(06)00309-9/ fulltext $>$. Acesso em: 25 maio, 2010. doi:10.1016/ j.jada.2006.03.010.

ULBRICHT, T.L.V.; SOUTHGATE, D.A.T. Coronary heart disease: seven dietary factors. Lancet, v.338, n.8773, p.985992, 1991. Disponível em:<http://www.sciencedirect.com/ science/article/pii/014067369191846M>. Acesso em: $15 \mathrm{dez}$. 2011. doi: http://dx.doi.org/10.1016/j.bbr.2011.03.031.

VERA, R. et al. Características químicas de amêndoas de barueiros (Dipteryx alata Vog.) de ocorrência natural no cerrado do estado de Goiás, BRASIL. Revista Brasileira de Fruticultura, v.31, n.1, p.112-118, 2009. Disponível em: <http://www.scielo.br/ s c i e lo.php? s c ript=s ci arttext \& pid = S 0100 29452009000100017>. Acesso em: 22 jan. 2010. doi: 10.1590/ S0100-29452009000100017.

WEATHERILL, A.R. et al. Saturated and polyunsaturated fatty acids reciprocally modulate dendritic cell functions mediated through TLR4. Journal of Immunology, v.174, n.9, p.53905397, 2005. Disponível em: <http://www.jimmunol.org/ content/174/9/5390.full>. Acesso em: 10 mar. 2010 . 